\title{
Simple and precise factorization of the Jarlskog invariant for neutrino oscillations in matter
}

\author{
Peter B. Denton ${ }^{1, *}$ and Stephen J. Parke ${ }^{2, \dagger}$ \\ ${ }^{1}$ Physics Department, Brookhaven National Laboratory, Upton, New York 11973, USA \\ ${ }^{2}$ Theoretical Physics Department, Fermi National Accelerator Laboratory, Batavia, IL 60510, USA
}

(Received 4 March 2019; revised manuscript received 20 June 2019; published 11 September 2019)

\begin{abstract}
For neutrino propagation in matter, we show that the Jarlskog invariant, which controls the size of true $C P$ violation in neutrino oscillation appearance experiments, factorizes into three pieces: the vacuum Jarlskog invariant times two simple two-flavor matter resonance factors that control the matter effects for the solar and atmospheric resonances independently. If the solar effective matter potential and the atmospheric effective $\Delta m^{2}$ are chosen carefully for these two resonance factors, then the fractional corrections to this factorization are an impressive $0.04 \%$ or smaller. We also show that the inverse of the square of the Jarlskog in matter $\left(1 / \hat{J}^{2}\right)$ is a fourth order polynomial in the matter potential which guarantees that it can be factored into two quadratics which immediately implies the functional form of our approximate, factorized expression.
\end{abstract}

DOI: 10.1103/PhysRevD.100.053004

\section{INTRODUCTION}

The discovery of an invariant, the Jarlskog invariant [1], that controls the size of $C P$ violation in both quark and lepton sectors was a monumental step in the understanding of flavor physics. For neutrinos, using the standard parametrization of the Pontecorvo-Maki-Nakagawa-Sakata matrix $[2,3]$, the Jarlskog invariant is given by

$$
J \equiv s_{13} c_{13}^{2} s_{12} c_{12} s_{23} c_{23} \sin \delta
$$

where we use the usual notation, $c_{i j}=\cos \theta_{i j}, s_{i j}=\sin \theta_{i j}$, and $\delta$ is the $C P$-violating phase. The $C P$-violating part of the vacuum neutrino oscillation probability in the appearance channels, e.g., $\nu_{\mu} \rightarrow \nu_{e}$, is given by [4]

$$
8 J \sin \Delta_{31} \sin \Delta_{32} \sin \Delta_{21}
$$

where the kinematic phases are given by $\Delta_{j k}=\Delta m_{j k}^{2} L / 4 E_{\nu}$ with $\Delta m_{j k}^{2}=m_{j}^{2}-m_{k}^{2}$ for an experiment of baseline $L$ and neutrino energy $E_{\nu}$.

For neutrinos propagating in matter, as in the NOvA [5], T2K [6], DUNE [7] and T2HK(K) [8,9] experiments, the

\footnotetext{
pdenton@bnl.gov

parke@fnal.gov
}

Published by the American Physical Society under the terms of the Creative Commons Attribution 4.0 International license. Further distribution of this work must maintain attribution to the author(s) and the published article's title, journal citation, and DOI. Funded by SCOAP ${ }^{3}$. part of the appearance oscillation probability that depends on the intrinsic $C P$ violation is given by

$$
8 \hat{J} \sin \hat{\Delta}_{31} \sin \hat{\Delta}_{32} \sin \hat{\Delta}_{21}
$$

where $\hat{x}$ is the matter value for the vacuum variable $x$. The Jarlskog invariant in matter, $\hat{J}$, is given by same expression as Eq. (1), but with the mixing angles and phase replaced by their matter values [10-12]. Both $\theta_{12}$ and $\theta_{13}$ have a strong dependence on density of the matter and the energy of the neutrino through the Wolfenstein matter potential [13], $a$, given by

$$
a \equiv 2 \sqrt{2} G_{F} N_{e} E_{\nu}
$$

where $G_{F}$ is the Fermi constant, $N_{e}$ is the number density of electrons, and $E_{\nu}$ is the neutrino energy in the matter rest frame.

\section{THE APPROXIMATE FACTORIZATION}

While the exact expressions for the mixing angles in matter are extremely complicated [10], it is possible to relate the Jarlskog invariant in matter to the vacuum Jarlskog, at the $0.04 \%$ level, as simply

$$
J \approx \mathcal{S}_{\odot} \mathcal{S}_{\mathrm{atm}} \hat{J}
$$

where 


$$
\begin{aligned}
\mathcal{S}_{\odot} & =\sqrt{\left(\cos 2 \theta_{12}-c_{13}^{2} a / \Delta m_{21}^{2}\right)^{2}+\sin ^{2} 2 \theta_{12}}, \\
\mathcal{S}_{\text {atm }} & =\sqrt{\left(\cos 2 \theta_{13}-a / \Delta m_{e e}^{2}\right)^{2}+\sin ^{2} 2 \theta_{13}} .
\end{aligned}
$$

Equation (5) shows simply how to relate the quantity measured in experiments, $\hat{J}$, to the amount of $C P$ violation in the lepton sector, $J$. The $\mathcal{S}$ factors are the two-flavor resonance factors associated with the solar and atmospheric resonances. Like the Jarlskog invariant, these $\mathcal{S}$ factors can also be written in a convention independent form, see Eq. (23).

The precision scales like $\mathcal{O}\left(s_{13}^{2} \cos 2 \theta_{12}\left(\Delta m_{21}^{2} / \Delta m_{e e}^{2}\right)\right)$ and $\mathcal{O}\left(s_{12}^{2} c_{12}^{2}\left(\Delta m_{21}^{2} / \Delta m_{e e}^{2}\right)^{2}\right)$ leading to an actual fractional precision of $\sim 0.04 \%$ for this factorization. To achieve this level of precision, we note that the following are crucial:

(i) for the solar (1-2) resonance factor, $\mathcal{S}_{\odot}$, the effective matter potential is $c_{13}^{2} a$, not just $a$,

(ii) for the atmospheric (1-3) resonance factor, $\mathcal{S}_{\text {atm }}$, the effective $\Delta m^{2}$ is $\Delta m_{e e}^{2} \equiv c_{12}^{2} \Delta m_{31}^{2}+s_{12}^{2} \Delta m_{32}^{2}$ $[14,15]$, not $\Delta m_{31(2)}^{2}$.

In Fig. 1, we have plotted the fractional precision to the approximation in Eq. (5) as a function of the matter potential for both neutrinos and antineutrinos and find that the expression is precise to the $0.04 \%$ level or better. We have numerically verified that $c_{13}^{2}$ is the optimal correction, $\Delta m_{e e}^{2}$ is the optimal atmospheric mass splitting, and that these results are generally independent of the mass ordering.

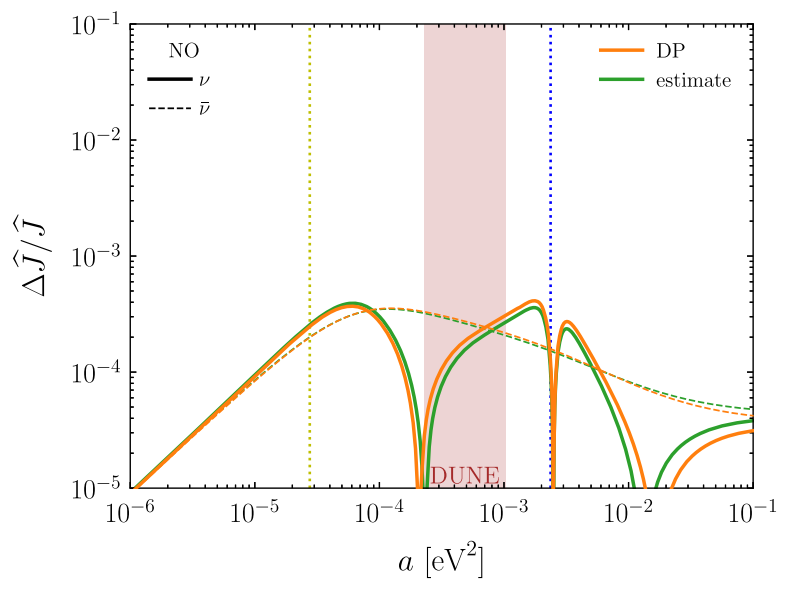

FIG. 1. The fractional precision in $\hat{J}$ compares our approximate expression with the exact expression calculated from [10], or Eqs. (15) and (16). The orange curves our approximate expression from Eqs. (5) and (6). The green curves are the analytic approximation of the precision shown in Eq. (19). The yellow and blue vertical lines are the solar and atmospheric resonances, respectively. The vertical strip is the amount of matter potential that DUNE will probe. The downward spikes occur where the exact and approximate expressions cross. The normal mass ordering (NO) is assumed.
Without the $c_{13}^{2}$ term in $\mathcal{S}_{\odot}$, or for different values of the solar matter potential, the precision is $2 \%-3 \%$ independent of the atmospheric mass splitting used in $\mathcal{S}_{\text {atm }}$. The precision is $\mathcal{O}\left(s_{13}^{2}\right)$ and $\mathcal{O}\left(\frac{\Delta m_{21}^{2}}{\Delta m_{e e}^{2}}\right)$, see the expression in Ref. [16]. With the $c_{13}^{2}$ term the precision is better, but still at the $2 \%$ level, although it is better in DUNE's region of interest, down to $\sim 0.1 \%-1 \%$, depending on which atmospheric $\Delta m^{2}$ is used in the $\mathcal{S}_{\text {atm }}$ term. When the atmospheric splitting is $\Delta m_{e e}^{2}$, the precision improves considerably down to the $0.04 \%$ level or better for any matter potential. That is, the precision is $\mathcal{O}\left(s_{13}^{2} \cos 2 \theta_{12}\left(\Delta m_{21}^{2} / \Delta m_{e e}^{2}\right)\right), \quad \mathcal{O}\left(s_{12}^{2} c_{12}^{2}\left(\Delta m_{21}^{2} / \Delta m_{e e}^{2}\right)^{2}\right)$, or better for all values of the matter potential. This is all shown in Fig. 2. Our result is the solid orange curve, $\left(e e, c_{13}^{2}\right)$ and the result from Ref. [16] which is the $(e e, 1)$ case is the solid blue curve.

We also note that this factorization is far from obvious from the context of angles as well. First, the $\theta_{23}$ and $\delta$ sector can be factored in a straightforward fashion by the Toshev identity [17]

$$
\sin 2 \hat{\theta}_{23} \sin \hat{\delta}=\sin 2 \theta_{23} \sin \delta .
$$

This statement is further enhanced by the fact that it is numerically known that $\hat{\theta_{23}}$ and $\hat{\delta}$ do not vary much in matter. After factoring out those two parameters, this leaves $s_{\widehat{13}} c_{\widehat{13}}^{2} s_{\widehat{12}} c_{\widehat{12}}=\hat{J} / s_{\widehat{23}} c_{\widehat{23}} \sin \hat{\delta}$. This term can be further factored into two terms where one is governed by the atmospheric mass splitting and the other by the solar mass splitting. For the atmospheric splitting, the factorization is simple

$$
s_{13} c_{\widehat{13}} \approx s_{13} c_{13} / \mathcal{S}_{\mathrm{atm}},
$$

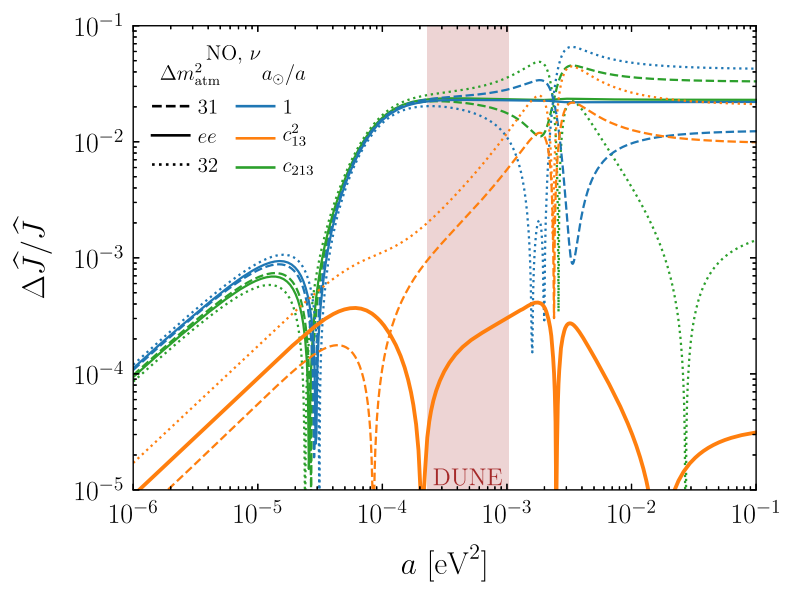

FIG. 2. The figure is the same as Fig. 1 for neutrinos only. We now vary the $\Delta m^{2}$ that appears in $\mathcal{S}_{\text {atm }}$ and the correction to the matter potential that appears in $\mathcal{S}_{\odot}$. Our solution is shown in the solid orange curve and is clearly the most precise in general and, in particular, for DUNE's region of interest. The result from [16] is the blue solid curve. 
Eq. (8), follows directly from the zeroth order approximation of Denton-Minakata-Parke (DMP) [18].

Counterintuitively, for the solar splitting

$$
c_{\widehat{13}} s_{12} c_{\widehat{12}} \approx c_{13} s_{12} c_{12} / \mathcal{S}_{\odot} .
$$

The extra $c_{\hat{13}}$ is required on the left-hand side (LHS) of Eq. (9) to give the LHS the same $a \rightarrow \infty$ limit as the righthand side (RHS). There is no direct analog in DMP for Eq. (9). Each of these approximations, Eqs. (8)-(9), are precise at the $0.4 \%$ level. However, when combined, there is a further cancellation and the product has a precision of $0.04 \%$. This returns us to our primary result, Eq. (5).

\section{UNDERSTANDING THE PRECISION}

In order to understand why Eqs. (5) and (6) achieve such precision and to estimate the precision in this factorization analytically, we use the exact Naumov-Harrison-Scott (NHS) identity [19,20],

$$
\hat{J} \Delta{\widehat{m^{2}}}_{32} \Delta{\widehat{m^{2}}}_{31} \Delta{\widehat{m^{2}}}_{21}=J \Delta m_{32}^{2} \Delta m_{31}^{2} \Delta m_{21}^{2},
$$

to rewrite the approximate factorization in terms of the exact matter eigenvalues,

$$
\Delta{\widehat{m^{2}}}_{32} \Delta{\widehat{m^{2}}}_{31} \Delta \widehat{m}_{21}^{2} \approx \mathcal{S}_{\odot} \mathcal{S}_{\mathrm{atm}} \Delta m_{32}^{2} \Delta m_{31}^{2} \Delta m_{21}^{2}
$$

While the exact eigenvalues have a very complicated analytic form [10] due to the presence of the $\cos \left(\frac{1}{3} \cos ^{-1} \ldots\right)$ terms, we have discovered that the square of the product of the difference of the eigenvalues in matter (also $1 / \hat{J}^{2}$ thanks to the NHS identity) can be written without any appearance of these $\cos \left(\frac{1}{3} \cos ^{-1} \cdots\right)$ factors, see Sec. IV below. In fact, $1 / \hat{J}^{2}$ can be written as a simple polynomial of the vacuum parameters and the matter potential. Since each of the $\widehat{\Delta m^{2}}$,s scale with the matter potential in some way as shown in Fig. 3, one would expect that the product of all three, squared, would be sixth order in the matter potential. In fact, the product of the three $\Delta \widehat{m^{2}}$, s squared is, in fact, only fourth order. This statement is independent of the hierarchical measured neutrino mass splittings. This suggests that only two matter corrections of the form given in Eq. (6) are needed and clearly justifies the form of Eq. (5).

Next, to understand the specific terms in $\mathcal{S}_{\text {atm }}$ and $\mathcal{S}_{\odot}$, we calculate various limits. First, consider the large matter potential limit, $a \rightarrow \infty$, then the eigenvalues are as follows:

$$
\begin{aligned}
& \widehat{m^{2}}{ }_{3} \rightarrow a+\Delta m_{e e}^{2} s_{13}^{2} \\
& \widehat{m^{2}}{ }_{2} \rightarrow \Delta m_{31}^{2} c_{13}^{2}\left(1+\mathcal{O}\left(\epsilon^{2} s_{12}^{2} c_{12}^{2}\right)\right) \\
& \widehat{m^{2}}{ }_{1} \rightarrow \Delta m_{21}^{2} c_{12}^{2}\left(1+\mathcal{O}\left(\epsilon^{2} s_{12}^{2} c_{12}^{2}\right)\right) .
\end{aligned}
$$

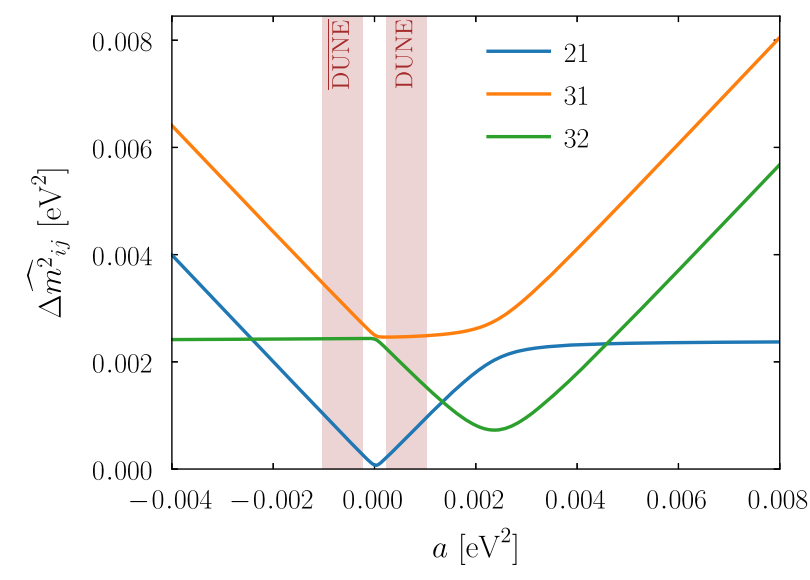

FIG. 3. The dependence of the three $\widehat{\Delta m^{2}}$, s in matter for the normal ordering. DUNE's region of interest for antineutrinos and neutrinos are shown in the shaded regions.

Thus the ${\widehat{\Delta m^{2}}}_{j k}$ are

${\widehat{\Delta m^{2}}}_{31} \approx a \approx \Delta m_{21}^{2} \mathcal{S}_{\odot} / c_{13}^{2}$

$\Delta \widehat{m}^{2}{ }_{32} \approx a \approx \Delta m_{e e}^{2} \mathcal{S}_{\mathrm{atm}}$

$\begin{aligned} \widehat{\Delta m^{2}}{ }_{21} \approx & c_{13}^{2}\left(\frac{\Delta m_{31}^{2} \Delta m_{32}^{2}}{\Delta m_{e e}^{2}}\right) \\ & \times\left(1+\mathcal{O}\left(\epsilon^{2} s_{12}^{2} c_{12}^{2}\right)+\mathcal{O}\left(\epsilon s_{13}^{2} \cos 2 \theta_{12}\right)\right) .\end{aligned}$

To understand why we associate $\widehat{\Delta m^{2}}{ }_{31} \approx \Delta m_{21}^{2} \mathcal{S}_{\odot} / c_{13}^{2}$ and $\widehat{\Delta m^{2}}{ }_{32} \approx \Delta m_{e e}^{2} \mathcal{S}_{\text {atm }}$ see [21]. Thus it is simple to see that

$$
\Delta \widehat{m}^{2}{ }_{32} \Delta \widehat{m}^{2}{ }_{31} \Delta \widehat{m^{2}}{ }_{21} \approx \mathcal{S}_{\odot} \mathcal{S}_{\mathrm{atm}} \Delta m_{32}^{2} \Delta m_{31}^{2} \Delta m_{21}^{2}
$$

in this limit. For this the factorization to work, it is crucial that the limit of

$$
{\widehat{\Delta m^{2}}}_{21} \approx c_{13}^{2}\left(\frac{\Delta m_{31}^{2} \Delta m_{32}^{2}}{\Delta m_{e e}^{2}}\right)
$$

This is where the appearance of the two essential factors, $c_{13}^{2}$ and $\Delta m_{e e}^{2}$, come from for this limit. What is highly nontrivial is that it is exactly these factors that are needed at the solar resonance and the atmospheric resonance, respectively.

Now consider a number of other simplifying limits: if $s_{13}^{2}=0$ and $s_{12}^{2}=0$ then it is trivial to show the factorization of Eq. (5) is exact. If $s_{13}^{2} \neq 0$ and $s_{12}^{2}=0$ then factional corrections to the factorization are $\epsilon s_{13}^{2}$ whereas if $s_{13}^{2}=0$ and $s_{12}^{2} \neq 0$ then fractional corrections are $\epsilon^{2} s_{12}^{2} c_{12}^{2}$, see Appendix A. 


\section{EXACT JARLSKOG INVARIANT IN MATTER}

For the general cases with $s_{13}^{2} \neq 0$ and $s_{12}^{2} \neq 0$, start from the cubic characteristic equation for the eigenvalues of the square of the neutrino masses in matter, $\widehat{m^{2}}{ }_{j}$, which each satisfy

$$
\left(\widehat{m}_{j}^{2}\right)^{3}-A\left({\widehat{m^{2}}}_{j}\right)^{2}+B{\widehat{m^{2}}}_{j}-C=0
$$

where $A, B$, and $C$ are the sum of the eigenvalues, sum of the products of the eigenvalues, and the triple product of the eigenvalues,

$$
\begin{aligned}
A & \equiv \sum_{j} \widehat{m^{2}}{ }_{j}=\Delta m_{31}^{2}+\Delta m_{21}^{2}+a, \\
B & \equiv \sum_{j>k} \widehat{m^{2}}{ }_{j} \widehat{m}^{2} \\
& =\Delta m_{31}^{2} \Delta m_{21}^{2}+a\left(\Delta m_{31}^{2} c_{13}^{2}+\Delta m_{21}^{2}\left(c_{12}^{2}+s_{13}^{2} s_{12}^{2}\right)\right), \\
C & \equiv \prod_{j} \widehat{m^{2}}{ }_{j}=a \Delta m_{31}^{2} \Delta m_{21}^{2} c_{13}^{2} c_{12}^{2},
\end{aligned}
$$

using the convention that $\left(\widehat{m^{2}}, \widehat{m^{2}}{ }_{2}, \widehat{m^{2}}{ }_{3}\right)=$ $\left(0, \Delta m_{21}^{2}, \Delta m_{31}^{2}\right)$ in vacuum.

Then it is straightforward to show that,

$$
\left(\prod_{j>k} \Delta{\widehat{m^{2}}}_{j k}\right)^{2}=\left(A^{2}-4 B\right)\left(B^{2}-4 A C\right)+(2 A B-27 C) C .
$$

This equation is a general identity for Eq. (14), independent of the exact values of $\mathrm{A}, \mathrm{B}, \mathrm{C}$, and is invariant under making the same shift of all of the eigenvalues, as it must given the LHS.

Combining Eqs. (15) and (16) one obtains the exact expression for $\left(\prod_{i>j} \Delta \widehat{m}_{i j}^{2}\right)^{2}$ as a fourth order polynomial in the matter potential, $a$. This guarantees its factorization in two quadratics as shown by Lodovico de Ferrari in 1540. To our knowledge this is the only exact measurable expression relating to neutrinos oscillating in matter without the $\cos \left(\frac{1}{3} \cos ^{-1} \ldots\right)$ term. ${ }^{1}$ This means that it is, in principle, possible to write the exact Jarlskog in matter in the same form as Eq. (5), however since the solutions to the

\footnotetext{
${ }^{1}$ In principle expressions like $A, B$, and $C$ in Eq. (15) are measurable as well, and they do not contain the $\cos \left(\frac{1}{3} \cos ^{-1} \cdots\right)$ term. In practice, measuring all three eigenvalues in the same matter effect is extremely difficult and not likely to occur even with future experiments. At the moment $\widehat{\Delta m^{2}}{ }_{21}$ is only measured in the sun while $\Delta \widehat{m}^{2}{ }_{31}$ and $\widehat{\Delta m^{2}}{ }_{32}$ are only measured in the Earth making a direct sum or product of these quantities in Eq. (15) not possible.
}

quartic expression which go in to Eq. (6) are a function of a cubic equation and are extremely complicated, the exact solution is far from simple. By leveraging the fact that we know that $s_{13}^{2}$ and $\Delta m_{21}^{2} / \Delta m_{e e}^{2}$ are small numbers, it is possible to drastically simplify that expression to one that is extremely compact.

\section{ERROR ESTIMATE}

Next, we compare the exact expression and our approximate expression and expand the difference in powers of $\epsilon$ and $s_{13}^{2}$. One can show that the correction $\Delta\left(\pi^{2}\right)$ is well approximated by the simple form,

$$
\begin{aligned}
&\left(\prod_{i>j} \Delta{\widehat{m^{2}}}_{i j}\right)^{2}=\mathcal{S}_{\odot}^{2} \mathcal{S}_{\mathrm{atm}}^{2}\left(\prod_{i>j} \Delta m_{i j}^{2}\right)^{2}+\Delta\left(\pi^{2}\right) \\
& \text { where } \Delta\left(\pi^{2}\right) \approx+2 \epsilon^{2} s_{13}^{2} a\left(\Delta m_{e e}^{2}\right)^{5} \\
&-2 \epsilon s_{13}^{2} \cos 2 \theta_{12} a^{2} \mathcal{S}_{\mathrm{atm}}^{2}\left(\Delta m_{e e}^{2}\right)^{4} \\
&-2 \epsilon^{2} s_{12}^{2} c_{12}^{2} a^{3}\left(\Delta m_{e e}^{2}-a\right)\left(\Delta m_{e e}^{2}\right)^{2} .
\end{aligned}
$$

This approximate expression for $\Delta\left(\pi^{2}\right)$ contains the first corrections to the factorization for each power of the matter potential. There is no constant term $\left(a^{0}\right)$ because the approximate expression is exact in vacuum, and there are only terms up to $a^{4}$ since both the exact and the approximate expressions only have terms up to $a^{4}$. An exact expression for $\Delta\left(\pi^{2}\right)$ can easily be obtained using Eqs. (16) and (15), see Appendix A. The fractional corrections are of $\mathcal{O}\left(\epsilon s_{13}^{2}\right)$ or $\mathcal{O}\left(\epsilon^{2} s_{12}^{2} c_{12}^{2}\right)$ for each power of $a$ and are of order a few $\times 10^{-4}$.

By propagating the correction from the product of $\widehat{\Delta m^{2}}$, squared to the correction in $\hat{J}$ via the Naumov-HarrisonScott identity, we find that the fractional precision in $\hat{J}$ is approximately given by

$$
\frac{\Delta \hat{J}}{\hat{J}} \approx \frac{\Delta\left(\pi^{2}\right)}{2 \mathcal{S}_{\odot}^{2} \mathcal{S}_{\mathrm{atm}}^{2}\left(\prod_{i>j} \Delta m_{i j}^{2}\right)^{2}}
$$

up to an overall sign. In Fig. 1 we plot Eq. (19) [note that using either the exact expression for the denominator or the approximate factorized expression given by Eq. (5) is indistinguishable]. Also shown for comparison is the exact fractional precision of Eqs. (5) and (6) as in Fig. 1. The agreement between the approximate, Eq. (19), and the exact fractional correction is excellent. We note that this precision estimate gets the magnitude of the precision correct as well as the general features: the precision goes to zero for small $a$ and peaks at the level of $0.04 \%$. In addition, the difference passes through zero for $a \approx \Delta m_{e e}^{2}$ for neutrinos but not for antineutrinos as reasonably expected due to the atmospheric resonance. 
To gain further insight, we explore the small and large matter potential limits. First we evaluate the precision below the solar resonance, $|a| \ll \Delta m_{21}^{2} \cos 2 \theta_{12}$, and above the atmospheric resonance, $|a| \gg\left|\Delta m_{e e}^{2}\right|$. Using the expression for $\Delta\left(\pi^{2}\right)$, Eq. (18), we find that in the low (high) $a$ limit we have that the fractional precision scales like

$$
\begin{aligned}
& \lim _{a \rightarrow 0} \frac{\Delta \hat{J}}{\hat{J}} \approx \epsilon s_{13}^{2} \frac{a}{\Delta m_{21}^{2}}=3 \times 10^{-4} \frac{a}{\Delta m_{21}^{2} \cos 2 \theta_{12}} \\
& \lim _{a \rightarrow \infty} \frac{\Delta \hat{J}}{\hat{J}} \approx \epsilon s_{13}^{2} \cos 2 \theta_{12}-\epsilon^{2} s_{12}^{2} c_{12}^{2}=6 \times 10^{-5},
\end{aligned}
$$

up to overall signs. It is interesting to note that the excellent precision at large $a$ is due to the significant cancellation in Eq. (21) that happens only for the NO. For the inverted ordering there is no cancellation since $\epsilon<0$ and the precision levels off at $a \gtrsim \Delta m_{e e}^{2}$ to $\Delta \hat{J} / \hat{J}=4 \times 10^{-4}$.

\section{DISCUSSION}

The Jarlskog invariant in vacuum can be written in a convention independent ${ }^{2}$ fashion,

$$
J=\Im\left(U_{e 1} U_{\mu 2} U_{e 2}^{*} U_{\mu 1}^{*}\right) .
$$

In addition, the Jarlskog in matter must also be convention independent due to the Naumov-Harrison-Scott identity. Therefore it must be the case that the approximate matter corrections $\mathcal{S}_{\odot, \text { atm }}$ can also be written in a convention independent way. These expressions are,

$$
\begin{aligned}
\mathcal{S}_{\odot}^{2} & =\left(1-\left(1-\left|U_{e 3}\right|^{2}\right) \frac{a}{\Delta m_{21}^{2}}\right)^{2}+4\left|U_{e 2}\right|^{2} \frac{a}{\Delta m_{21}^{2}}, \\
\mathcal{S}_{\mathrm{atm}}^{2} & =\left(1-\frac{a}{\Delta m_{e e}^{2}}\right)^{2}+4\left|U_{e 3}\right|^{2} \frac{a}{\Delta m_{e e}^{2}},
\end{aligned}
$$

which are essentially as simple as those with mixing angles in Eq. (6). See Appendix B for alternative ways to write these resonance factors. We can similarly write the atmospheric mass splitting as

$$
\Delta m_{e e}^{2}=\Delta m_{31}^{2}-\frac{\left|U_{e 2}\right|^{2}}{1-\left|U_{e 3}\right|^{2}} \Delta m_{21}^{2}
$$

where $\left|U_{e 3}\right|^{2} \approx 0.022$ and $\left|U_{e 2}\right|^{2} \approx 0.31$ and unitarity implies $\left|U_{e 1}\right|^{2}=1-\left|U_{e 2}\right|^{2}-\left|U_{e 3}\right|^{2}$. It is natural to associate the correction for the atmospheric resonance factor on the $\Delta m^{2}$ as the minimum separation is $\Delta m_{e e}^{2} \sin 2 \theta_{13}$, whereas for the solar resonance factor, since the minimum

\footnotetext{
${ }^{2}$ By convention independent, we mean regardless of how one parametrizes the lepton mixing matrix - that is, without any reference to the mixing angles.
}

separation is not altered at $\Delta m_{21}^{2} \sin 2 \theta_{12}$, it is natural to associate the correction with the matter potential.

In light of the Naumov-Harrison-Scott identity, it is not surprising that $\hat{J} / J$ has a form that looks like the inverse of several matter corrections to the $\Delta m^{2}$ 's. It may not be obvious, however, why $\hat{J}$ is well-approximated by only two such expressions instead of all three. The reason is because for nearly any value of $a$, there is always one $\widehat{\Delta m^{2}}$ that is

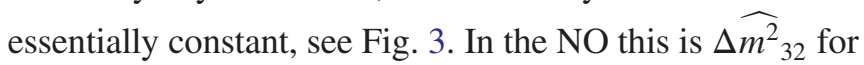
antineutrinos and $\widehat{\Delta m_{\ell 1}^{2}}$ for neutrinos where $\ell=3$ below the atmospheric resonance and $\ell=2$ above the atmospheric resonance. As such having two $\mathcal{S}$ terms is to be expected. This fact is further reinforced by the fact that the exact expression for the square of the product of the $\widehat{\Delta m^{2}}{ }_{i j}$ is fourth order in $a$, which directly proves that only two $\Delta \widehat{m}^{2}{ }_{i j}$ require a matter correction. Moreover, we expect that, when squared, that correction should be fourth order in the matter potential, as is the case for $\mathcal{S}_{\text {atm }}^{2} \mathcal{S}_{\odot}^{2}$.

In addition, while the presence of the $c_{13}^{2}$ term breaks an otherwise relatively symmetric definition of $\mathcal{S}_{\odot}$ and $\mathcal{S}_{\text {atm }}$, this can be understood using the DMP [18] expressions. In that formalism the (1-2) sector is handled second and thus contains a small (1-3) correction since the (1-3) sector was handled first.

We also note that the functional form of the $\mathcal{S}$ functions have shown up elsewhere in the literature [16,21-24] with slight differences. One interesting example is the quantity $\Delta m_{e e}^{2} \mathcal{S}_{\text {atm }}$ [using the same definition as in Eq. (6)] which was shown [21] to be an excellent approximation for the frequency of $\nu_{e}$ disappearance in matter, i.e., $\Delta m_{e e}^{2}$ in matter.

It is useful to consider the level of precision in a broader phenomenological context. Since DUNE is striving to reach percent level precision, it is clear that $2 \%$ precision, such as that reached in Ref. [16] is not sufficient, thus precision at the $0.04 \%$ level is necessary to be precise enough for DUNE. On the other hand, there are unavoidable uncertainties in the oscillation probabilities due to uncertainties in the matter density. A $1 \%$ overall uncertainty in the matter density profile results in a $0.1 \%$ fractional uncertainty in the oscillation probability. Alternatively, by considering various density profiles available [25], the shape uncertainty can be estimated, and is found to be only $0.01 \%$ [26]. Thus precision at the $0.04 \%$ level is also a sufficient level of precision.

Finally, we recall the exact Toshev identity, $\sin 2 \hat{\theta_{23}} \sin \hat{\delta}=\sin 2 \theta_{23} \sin \delta$ [17]. Thus, in terms of the mixing angles, the ratio $\hat{J} / J$ has no dependence (explicit or otherwise) on $\theta_{23}$ or $\delta$. This fact is approximately confirmed by the fact that $\mathcal{S}_{\text {atm }}$ and $\mathcal{S}_{\odot}$ have no dependence on $\theta_{23}$ and $\delta$. This brings to attention an important point on $C P$ violation: the quantity that describes the amount of $C P$ violation in the lepton sector is the Jarlskog invariant, $J$, not 
the $C P$-violating phase, $\delta$, and thus it is the Jarlskog that should be reported by experiments as a measure of the amount of $C P$ violation. Since DUNE will measure each part of the vacuum Jarlskog in the atmospheric sector, $\theta_{23}$, $\theta_{13}$, and $\delta$ and could measure $\theta_{12}$ using solar neutrinos as well [27], it is, in principle, possible to completely determine the Jarlskog in the lepton sector with a single experiment.

\section{CONCLUSIONS}

In this paper we have shown that the Jarlskog invariant in the lepton sector is exactly equal to the Jarlskog in matter times two matter resonance factors. While the coefficients of $a$ and $a^{2}$ in these resonances are extremely complicated solutions to a quartic equation, they can be simplified immensely while still retaining precision at the $\sim 0.04 \%$, i.e., $\mathcal{O}\left(s_{13}^{2} \cos 2 \theta_{12}\left(\Delta m_{21}^{2} / \Delta m_{e e}^{2}\right)\right)$ and $\mathcal{O}\left(s_{12}^{2} c_{12}^{2}\left(\Delta m_{21}^{2} / \Delta m_{e e}^{2}\right)^{2}\right)$, a level of precision that is both necessary and sufficient for future long-baseline experiments.

We have also derived the exact Jarlskog invariant in matter as a simple fourth order polynomial in the matter potential which allows us to estimate analytically the precision of the factorization. To achieve the high precision given here, it is crucial to use the $\theta_{13}$ corrected value for the matter potential for the solar (1-2) sector, $a c_{13}^{2}$ as well as the effective $\Delta m_{e e}^{2}$ instead of $\Delta m_{31}^{2}$ or $\Delta m_{32}^{2}$ for the atmospheric (1-3) sector. This precision factorization of the Jarlskog invariant in matter further enhances our understanding of neutrinos in matter relevant for the currently running NOvA and T2K experiments and the upcoming DUNE and T2HK(K).

\section{ACKNOWLEDGMENTS}

P. B. D. acknowledges the United States Department of Energy under Grant Contract No. desc0012704. Fermilab is operated by the Fermi Research Alliance under Contract No. DE-AC02-07CH11359 with the U.S. Department of Energy. S. P. thanks IFT of Madrid for wonderful hospitality that inspired this work. This project has received funding/ support from the European Union's Horizon 2020 research and innovation programme under the Marie SklodowskaCurie Grant Agreement No. 690575 and No. 674896.

\section{APPENDIX A: EXACT DERIVATION}

First consider the case when $s_{13}^{2}=0$ with nonzero $s_{12}^{2}$, then it is easy to show that

$$
\begin{aligned}
\left(\prod_{i>j} \Delta{\widehat{m^{2}}}_{i j}\right)^{2}= & \left(\left(\Delta m_{21}^{2}\right)^{2}-2 a \Delta m_{21}^{2} \cos 2 \theta_{12}+a^{2}\right) \\
& \times\left(\Delta m_{31}^{2} \Delta m_{32}^{2}-a\left(\Delta m_{31}^{2}-\Delta m_{21}^{2} c_{12}^{2}\right)\right)^{2}
\end{aligned}
$$

exactly. To understand how this result relates to the factorization given by Eq. (5), one needs the following exact identity

$\left(\Delta m_{31}^{2}-\Delta m_{21}^{2} c_{12}^{2}\right)=\frac{\Delta m_{31}^{2} \Delta m_{32}^{2}+\left(\Delta m_{21}^{2}\right)^{2} s_{12}^{2} c_{12}^{2}}{\Delta m_{e e}^{2}}$,

where we note that $\Delta m_{31}^{2}-\Delta m_{21}^{2} c_{12}^{2}=\left(\Delta m_{\mu \mu}^{2}+\Delta m_{\tau \tau}^{2}\right) / 2$ of Ref. [14]. If one drops the $\left(\Delta m_{21}^{2}\right)^{2}$ terms in this identity then one recovers the $s_{13}^{2}=0$ limit of the factorization, Eq. (5). As we will see, all of the $\epsilon^{2} s_{12}^{2} c_{12}^{2}$ corrections come from this identity. In principle one could absorb the second term in this identity into the definition of $\Delta m_{e e}^{2}$ and remove such corrections.

For the case when $s_{12}^{2}=0$ and nonzero $s_{13}^{2}$, one can show that without approximation that

$$
\begin{aligned}
\left(\prod_{i>j} \Delta{\widehat{m^{2}}}_{i j}\right)^{2}= & \left(\left(\Delta m_{e e}^{2}\right)^{2}-2 a \Delta m_{e e}^{2} \cos 2 \theta_{13}+a^{2}\right) \\
& \times\left(\Delta m_{21}^{2} \Delta m_{32}^{2}-a\left(c_{13}^{2} \Delta m_{32}^{2}-s_{13}^{2} \Delta m_{21}^{2}\right)\right)^{2}
\end{aligned}
$$

In this limit $\Delta m_{e e}^{2}=\Delta m_{31}^{2}$, so that the factorization of Eq. (5) is recovered, if the $a s_{13}^{2} \Delta m_{21}^{2}$ term is set to zero.

Finally, one can show that when $s_{13}^{2} \neq 0$ and $s_{12}^{2} \neq 0$, the most general case, that

$$
\begin{aligned}
\left(\prod_{i>j} \Delta{\widehat{m^{2}}}_{i j}\right)^{2}= & \left(\left(\Delta m_{21}^{2}\right)^{2}-2 a \Delta m_{21}^{2} c_{13}^{2} \cos 2 \theta_{12}+a^{2} c_{13}^{4}\right) \\
& \times\left(\left(\Delta m_{31}^{2} \Delta m_{32}^{2}\right)^{2}-2 a\left(\Delta m_{31}^{2} \Delta m_{32}^{2}\right)\right. \\
& \times\left(\Delta m_{31}^{2}-\Delta m_{21}^{2} c_{12}^{2}\right) \cos 2 \theta_{13} \\
& \left.+a^{2}\left(\Delta m_{31}^{2}-\Delta m_{21}^{2} c_{12}^{2}\right)^{2}\right) \\
& +\left(s_{13}^{2} \Delta m_{21}^{2}\right)\left(\sum_{n=1}^{4} a^{n} P_{n}\right)
\end{aligned}
$$

where

$$
\begin{aligned}
P_{1}= & 2 \Delta m_{21}^{2} \Delta m_{31}^{2} \Delta m_{32}^{2} \Delta m_{e e}^{2} \\
P_{2}= & -2 \Delta m_{31}^{2} \Delta m_{32}^{2} \Delta m_{e e}^{2} c_{13}^{2} \cos 2 \theta_{12} \\
& -4 \Delta m_{31}^{2} \Delta m_{32}^{2} \Delta m_{21}^{2}\left(\cos 2 \theta_{13}-c_{13}^{2} s_{12}^{2} c_{12}^{2}\right) \\
& +\left(\Delta m_{e e}^{2}\right)^{2} \Delta m_{21}^{2} s_{13}^{2}+2\left(\Delta m_{21}^{2}\right)^{3} s_{12}^{2} c_{12}^{2} \\
P_{3}= & 2\left(\Delta m_{31}^{2} \Delta m_{32}^{2}+\left(\Delta m_{e e}^{2}\right)^{2}\right) c_{13}^{2} \cos 2 \theta_{12} \cos 2 \theta_{13} \\
& +4 \Delta m_{e e}^{2} \Delta m_{21}^{2}\left(s_{13}^{2}+s_{12}^{2} c_{12}^{2} c_{13}^{4}\right) \\
& -2\left(\Delta m_{21}^{2}\right)^{2} \cos 2 \theta_{12}\left(1+2 s_{12}^{2} c_{12}^{2} c_{13}^{2}\right) \\
P_{4}= & -\left(\Delta m_{31}^{2}+\Delta m_{32}^{2}\right) c_{13}^{2} \cos 2 \theta_{12}+\Delta m_{21}^{2} .
\end{aligned}
$$


Note, that all the corrections to the factorization here are proportional to $s_{13}^{2} \Delta m_{21}^{2} . \Delta\left(\pi^{2}\right)$ of Eq. (18) can now be trivially derived from Eqs. (A4) and (A2), (A5)-(A8), as well as the higher order corrections to $\Delta\left(\pi^{2}\right)$.

The zeros of $\left(\prod_{i>j} \Delta \hat{m}^{2}{ }_{i j}\right)^{2}$, in the complex matter potential plane, can also be obtained, for both the exact expression and our approximate factorized expression. Given the exact expressions for the zeros (which are extremely complicated and involve solving a general quartic equation which, in turn, involves solving a general cubic equation) it is possible, in principle, to write an exact expression of the form of Eqs. (5) and (6) with different functions of the vacuum parameters in the $\mathcal{S}_{\text {atm }}$ and $\mathcal{S}_{\odot}$ functions.

For the approximate expression, the zeros occur at

$a=\left(\Delta m_{21}^{2} / c_{13}^{2}\right) e^{ \pm i 2 \theta_{12}} \quad$ and $\quad a=\Delta m_{e e}^{2} e^{ \pm i 2 \theta_{13}}$.

Note the appearance of both $c_{13}^{2}$ and $\Delta m_{e e}^{2}$ in the location of these zeros. Without these exact factors the positions of the zeros move by $1 \%-2 \%$. For the exact expression, we have numerically calculated the location of the zeros in the complex matter potential plane and find that the fractional corrections to their position, compared to (A9), is

$$
6 \times 10^{-4} \text { and } 2 \times 10^{-4},
$$

for the solar and atmospheric zeros, respectively. For any polynomial, the location of the zeros determines the polynomial exactly, up to an overall factor, therefore the above, again, points to the necessity of including both $c_{13}^{2}$ and $\Delta m_{e e}^{2}$ in the factorization.

\section{APPENDIX B: ALTERNATIVE EXPRESSIONS FOR THE RESONANCE FACTORS}

\begin{abstract}
Alternatively, $\mathcal{S}_{\odot}$ can also be written as $\sqrt{1-2 \cos 2 \theta_{12}\left(c_{13}^{2} a / \Delta m_{21}^{2}\right)+\left(c_{13}^{2} a / \Delta m_{21}^{2}\right)^{2}} \quad$ and $\sqrt{\left(1-c_{13}^{2} a / \Delta m_{21}^{2}\right)^{2}+4 s_{12}^{2}\left(c_{13}^{2} a / \Delta m_{21}^{2}\right)}$ and similarly for $\mathcal{S}_{\text {atm }}$. Like the Jarlskog invariant, these $\mathcal{S}$ factors can also be written in a convention independent form, see Eq. (23). They can also be written as $\left|e^{2 i \theta_{12}}-c_{13}^{2} a / \Delta m_{21}^{2}\right|$ and similarly for $\mathcal{S}_{\text {atm }}$ which shows where the complex zeros are, see Appendix A.
\end{abstract}

[1] C. Jarlskog, Phys. Rev. Lett. 55, 1039 (1985).

[2] Z. Maki, M. Nakagawa, and S. Sakata, Prog. Theor. Phys. 28, 870 (1962).

[3] B. Pontecorvo, Zh. Eksp. Teor. Fiz. 53, 1717 (1967) [Sov. Phys. JETP 26, 984 (1968)].

[4] S. M. Bilenky and S. T. Petcov, Rev. Mod. Phys. 59, 671 (1987); 60, 575(E) (1988).

[5] D. S. Ayres et al. (NOvA Collaboration), arXiv:hep-ex/ 0503053.

[6] Y. Itow et al. (T2K Collaboration), in Neutrino Oscillations and their Origin. Proceedings, 3rd International Workshop, NOON 2001, Kashiwa, Tokyo, Japan (2001), pp. 239-248.

[7] R. Acciarri et al. (DUNE Collaboration), arXiv:1601.05471.

[8] K. Abe et al. (Hyper-Kamiokande Working Group), arXiv: 1412.4673 .

[9] K. Abe et al. (Hyper-Kamiokande Collaboration), Prog. Theor. Exp. Phys. 2018, 063 C01 (2018).

[10] H. W. Zaglauer and K. H. Schwarzer, Z. Phys. C 40, 273 (1988).

[11] P. I. Krastev and S. T. Petcov, Phys. Lett. B 205, 84 (1988).

[12] S. J. Parke and T. J. Weiler, Phys. Lett. B 501, 106 (2001).

[13] L. Wolfenstein, Phys. Rev. D 17, 2369 (1978).
[14] H. Nunokawa, S. J. Parke, and R. Zukanovich Funchal, Phys. Rev. D 72, 013009 (2005).

[15] S. Parke, Phys. Rev. D 93, 053008 (2016).

[16] X. Wang and S. Zhou, J. High Energy Phys. 05 (2019) 035.

[17] S. Toshev, Mod. Phys. Lett. A 06, 455 (1991).

[18] P. B. Denton, H. Minakata, and S. J. Parke, J. High Energy Phys. 06 (2016) 051.

[19] V. A. Naumov, Int. J. Mod. Phys. D 01, 379 (1992).

[20] P. F. Harrison and W. G. Scott, Phys. Lett. B 476, 349 (2000).

[21] P. B. Denton and S. J. Parke, Phys. Rev. D 98, 093001 (2018).

[22] M. Freund, Phys. Rev. D 64, 053003 (2001).

[23] E. K. Akhmedov, R. Johansson, M. Lindner, T. Ohlsson, and T. Schwetz, J. High Energy Phys. 04 (2004) 078.

[24] H. Minakata and S. J. Parke, J. High Energy Phys. 01 (2016) 180.

[25] B. Roe, Phys. Rev. D 95, 113004 (2017).

[26] K. J. Kelly and S. J. Parke, Phys. Rev. D 98, 015025 (2018).

[27] F. Capozzi, S. W. Li, G. Zhu, and J. F. Beacom, arXiv: 1808.08232 [Phys. Rev. Lett. (to be published)]. 